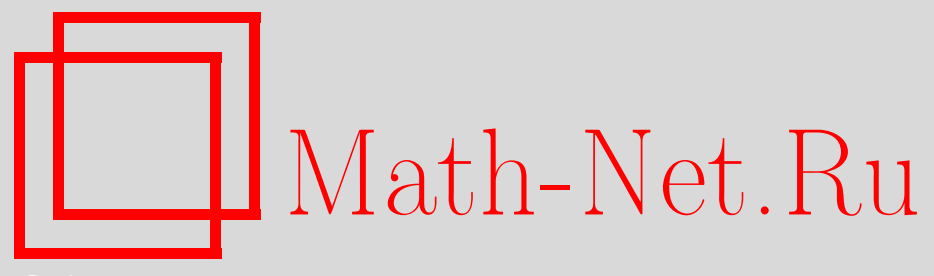

С. З. Пакуляк, С. М. Хорошкин, Метод проекций и универсальная весовая функция для квантовой аффинной алгебры $U_{q}\left(\widehat{\mathfrak{s l}}_{N+1}\right), T M \Phi, 2007$, том 150, номер 2, 286-303

DOI: https://doi.org/10.4213/tmf5979

Использование Общероссийского математического портала Math-Net.Ru подразумевает, что вы прочитали и согласны с пользовательским соглашением http://www . mathnet.ru/rus/agreement

Параметры загрузки:

IP : 18.209 .158 .208

26 апреля 2023 г., 17:07:09

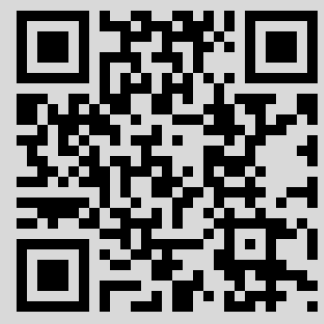




\title{
МЕТОД ПРОЕКЦИЙ И УНИВЕРСАЛЬНАЯ ВЕСОВАЯ ФУНКЦИЯ ДЛЯ КВАНТОВОЙ АФФИННОЙ АЛГЕБРЫ $U_{q}\left(\widehat{\mathfrak{s l}}_{N+1}\right)$
}

\begin{abstract}
Вычислена проекция произведений токов Дринфельда на пересечение различных борелевских подалгебр в токовой реализации квантовой аффинной алгебры $U_{q}\left(\widehat{\mathfrak{s l}}_{N+1}\right)$. Эта проекция задает универсальную весовую функцию и имеет структуру иерархических векторов Бете.
\end{abstract}

Ключевые слова: квантовые аффинные алгебры, иерархический анзац Бете.

\section{1. ВВЕДЕНИЕ}

Иерархические векторы Бете были введены в работе [1]. Они были построены для обобщения алгебраического анзаца Бете (развитого сначала для моделей, обладающих симметрией алгебры $\left.U_{q}\left(\widehat{\mathfrak{g l}}_{2}\right)\right)$ на интегрируемые модели, которые связаны с другими квантовыми аффинными алгебрами. Слово "иерархический” означает, что проблема выражения векторов Бете через матричные элементы матрицы монодромии (через произведение операторов $\prod_{i} B\left(\lambda_{i}\right)$ в случае моделей, связанных с алгеброй $\left.U_{q}\left(\widehat{\mathfrak{g l}}_{2}\right)\right)$ редуцируется к аналогичной задаче для алгебры меньшего ранга. Для получения собственных векторов коммутирующих интегралов движения из векторов Бете необходимо, чтобы параметры этих векторов $\left(\lambda_{i}\right.$ в произведении $\left.\prod_{i} B\left(\lambda_{i}\right)\right)$ удовлетворяли уравнениям Бете. Для моделей, связанных с алгеброй $U_{q}\left(\widehat{\mathfrak{g l}}_{N+1}\right)$, эта программа была реализована в работе [2]. Построение векторов Бете для других алгебр было осуществлено только в отдельных случаях.

Векторы Бете имеют широкое применение в теории уравнений Книжника-Замолодчикова (K3) [3], где они были использованы для построения интегральных решений этих уравнений. В теории К3, ассоциированной с алгеброй $\mathfrak{g}$, векторы Бете

* Институт теоретической и экспериментальной физики, Москва, Россия.

E-mail: khor@itep.ru

†Объединенный институт ядерных исследований, Дубна, Московская обл. Россия. E-mail: pakuliak@theor.jinr.ru 
(которые в этой теории называются весовыми функциями) являются векторнозначными функциями в тензорном произведении представлений старшего веса алгебры $U_{q}(\widehat{\mathfrak{g}})$. В работе [4] было показано, что весовые функции для алгебры $U_{q}\left(\widehat{\mathfrak{g l}}_{N+1}\right)$ удовлетворяют некоторым коалгебраическим свойствам. Там же была предъявлена конструкция весовой функции как некоторого матричного элемента $U_{q}\left(\widehat{\mathfrak{g l}}_{N+1}\right)$ монодромии.

Альтернативный подход к построению весовых функций был сформулирован в работе [5] для произвольной нескрученной квантовой аффинной алгебры. Эта конструкция использовала токовую реализацию Дринфельда [6] для квантовых аффинных алгебр и метод проекций [7], [8] на пересечения борелевских алгебр различного типа. Универсальная весовая функция была определена в работах [5], [9], [10] как проекция от произведений токов. Целью настоящей работы является вычисление проекции от произведений токов Дринфельда для квантовой аффинной алгебры $U_{q}\left(\widehat{\mathfrak{s l}}_{N+1}\right)$. Результат вычислений дает представление векторов Бете через токовые генераторы квантовой аффинной алгебры $U_{q}\left(\widehat{\mathfrak{s l}}_{N+1}\right)$.

Работа организована следующим образом. В разделе 2 сформулирована токовая реализация алгебры $U_{q}\left(\widehat{\mathfrak{s l}}_{N+1}\right)$ и описаны ее борелевские подалгебры различных типов. В разделе 3 сформулированы основные результаты работы. В разделе 4 приведены доказательства основных утверждений. Техническое утверждение, необходимое для основных доказательств, сформулировано и доказано в приложении.

\section{2. КВАНТОВАЯ АФФИННАЯ АЛГЕБРА $U_{q}\left(\widehat{\mathfrak{s l}}_{N+1}\right)$}

Квантовая аффинная алгебра $U_{q}\left(\widehat{\mathfrak{s l}}_{N+1}\right)$ имеет несколько реализаций. Первая реализация, которую мы назовем стандартной, использует генераторы Шевалле [11]. В этом описании алгебра $U_{q}\left(\widehat{\mathfrak{s l}}_{N+1}\right)$ образована генераторами $e_{ \pm \alpha_{i}}, k_{\alpha_{i}}^{ \pm 1}$, где $i=0,1, \ldots, N$ и $\prod_{i=0}^{N} k_{\alpha_{i}}=q^{c}=1^{1)}$, удовлетворяющими соотношениям

$$
\begin{gathered}
k_{\alpha_{i}} e_{ \pm \alpha_{j}} k_{\alpha_{i}}^{-1}=q^{ \pm a_{i j}} e_{ \pm \alpha_{j}}, \quad\left[e_{\alpha_{i}}, e_{-\alpha_{j}}\right]=\delta_{i j} \frac{k_{\alpha_{i}}-k_{\alpha_{i}}^{-1}}{q_{i}-q_{i}^{-1}} \\
\sum_{r=0}^{m_{i, j}} e_{ \pm \alpha_{i}}^{(r)} e_{ \pm \alpha_{j}} e_{ \pm \alpha_{i}}^{\left(m_{i, j}-r\right)}=0, \quad \text { где } \quad m_{i, j}=1-\left(\alpha_{i}, \alpha_{j}\right), \quad i \neq j \\
e_{ \pm \alpha_{i}}^{(r)}=\frac{e_{ \pm \alpha_{i}}^{r}}{[k]_{q} !}, \quad[k]_{q} !=[k]_{q}[k-1]_{q} \ldots[2]_{1}[1]_{q}, \quad[k]_{q}=\frac{q^{k}-q^{-k}}{q-q^{-1}}
\end{gathered}
$$

причем матрица с элементами $\left(\alpha_{i}, \alpha_{j}\right), i, j=0,1, \ldots, N$, является матрицей Картана аффинной алгебры $\widehat{\mathfrak{s l}}_{N+1},\left(\alpha_{i}, \alpha_{i}\right)=2,\left(\alpha_{i}, \alpha_{j}\right)=-1$, если $i-j= \pm 1(\bmod (N+1))$. Алгебра $U_{q}\left(\widehat{\mathfrak{s l}}_{N+1}\right)$ - это алгебра Хопфа, снабженная отображениями коумножения, антипода и коединицы. Коумножение на генераторах задано формулами

$$
\Delta\left(e_{\alpha_{i}}\right)=e_{\alpha_{i}} \otimes 1+k_{\alpha_{i}} \otimes e_{\alpha_{i}}, \quad \Delta\left(e_{-\alpha_{i}}\right)=1 \otimes e_{-\alpha_{i}}+e_{-\alpha_{i}} \otimes k_{\alpha_{i}}^{-1}, \quad \Delta\left(k_{\alpha_{i}}\right)=k_{\alpha_{i}} \otimes k_{i} .
$$

\footnotetext{
1) В настоящей работе алгебра $U_{q}\left(\widehat{\mathfrak{s l}}_{N+1}\right)$ рассматривается при нулевом значении центрального заряда, $c=0$, и при опущенном операторе градуировки.
} 
Вторая реализация, которую мы будем в основном использовать в этой работе, называется токовой реализацией. Она была введена в работе [6]. В этой реализации алгебра $U_{q}\left(\widehat{\mathfrak{s l}}_{N+1}\right)$ (вновь без оператора градуировки и при нулевом значении центрального заряда) образована элементами $e_{i}[n], f_{i}[n](n \in \mathbb{Z})$ и $\psi_{i}^{ \pm}[n](n \geqslant 0)$, $\psi_{i}^{+}[0]=\left(\psi_{i}^{-}[0]\right)^{-1}=k_{i}, i=1, \ldots, N$. Они могут быть собраны в производящие функции

$$
e_{i}(z)=\sum_{n \in \mathbb{Z}} e_{i}[n] z^{-n}, \quad f_{i}(z)=\sum_{n \in \mathbb{Z}} f_{i}[n] z^{-n}, \quad \psi_{i}^{ \pm}(z)=\sum_{n \geqslant 0} \psi_{i}^{ \pm}[n] z^{\mp n}
$$

удовлетворяющие следующим соотношениям:

$$
\begin{gathered}
\left(z-q^{\left(\alpha_{i}, \alpha_{j}\right)} w\right) e_{i}(z) e_{j}(w)=e_{j}(w) e_{i}(z)\left(q^{\left(\alpha_{i}, \alpha_{j}\right)} z-w\right), \\
\left(z-q^{-\left(\alpha_{i}, \alpha_{j}\right)} w\right) f_{i}(z) f_{j}(w)=f_{j}(w) f_{i}(z)\left(q^{-\left(\alpha_{i}, \alpha_{j}\right)} z-w\right), \\
\psi_{i}^{ \pm}(z) e_{j}(w)\left(\psi_{i}^{ \pm}(z)\right)^{-1}=\frac{q^{\left(\alpha_{i}, \alpha_{j}\right)} z-w}{z-q^{\left(\alpha_{i}, \alpha_{j}\right)} w} e_{j}(w), \\
\psi_{i}^{ \pm}(z) f_{j}(w)\left(\psi_{i}^{ \pm}(z)\right)^{-1}=\frac{q^{-\left(\alpha_{i}, \alpha_{j}\right)} z-w}{z-q^{-\left(\alpha_{i}, \alpha_{j}\right)} w} f_{j}(w), \\
\psi_{i}^{\mu}(z) \psi_{j}^{\nu}(w)=\psi_{j}^{\nu}(w) \psi_{i}^{\mu}(z), \quad \mu, \nu= \pm, \\
{\left[e_{i}(z), f_{j}(w)\right]=\frac{\delta_{i j} \delta(z / w)}{q-q^{-1}}\left(\psi_{i}^{+}(z)-\psi_{i}^{-}(w)\right),}
\end{gathered}
$$

а также соотношениям Серра

$$
\begin{aligned}
& \underset{z_{1}, z_{2}}{\operatorname{Sym}}\left(e_{i}\left(z_{1}\right) e_{i}\left(z_{2}\right) e_{j}(w)-\left(q+q^{-1}\right) e_{i}\left(z_{1}\right) e_{j}(w) e_{i}\left(z_{2}\right)+e_{j}(w) e_{i}\left(z_{1}\right) e_{i}\left(z_{2}\right)\right)=0, \\
& \operatorname{Sym}_{z_{1}, z_{2}}\left(f_{i}\left(z_{1}\right) f_{i}\left(z_{2}\right) f_{j}(w)-\left(q+q^{-1}\right) f_{i}\left(z_{1}\right) f_{j}(w) f_{i}\left(z_{2}\right)+f_{j}(w) f_{i}\left(z_{1}\right) f_{i}\left(z_{2}\right)\right)=0,
\end{aligned}
$$

где $i-j= \pm 1$. Структура коумножения $\Delta^{(\mathrm{D})}$ в алгебре $U_{q}\left(\widehat{\mathfrak{s l}}_{N+1}\right)$, которая естественным образом ассоциированна с токовой реализацией, задана в терминах токов:

$$
\begin{aligned}
\Delta^{(\mathrm{D})} e_{i}(z) & =e_{i}(z) \otimes 1+\psi_{i}^{-}(z) \otimes e_{i}(z), \\
\Delta^{(\mathrm{D})} f_{i}(z) & =1 \otimes f_{i}(z)+f_{i}(z) \otimes \psi_{i}^{+}(z), \\
\Delta^{(\mathrm{D})} \psi_{i}^{ \pm}(z) & =\psi_{i}^{ \pm}(z) \otimes \psi_{i}^{ \pm}(z) .
\end{aligned}
$$

Будем называть эту структуру токовой структурой Хопфа. Стандартное коумножение $\Delta$ и токовое коумножение $\Delta^{(\mathrm{D})}$ связаны преобразованием твиста, который может быть описан явно [12].

Переход от стандартной реализации $U_{q}\left(\widehat{\mathfrak{s l}}_{N+1}\right)$ к токовой осуществляется путем построения базиса Картана-Вейля [12]. В обратную сторону изоморфизм между 
двумя реализациями устанавливается с помощью отождествления

$$
\begin{aligned}
k_{\alpha_{i}}^{ \pm 1} \mapsto k_{i}^{ \pm 1}, \quad e_{\alpha_{i}} \mapsto e_{i}[0], \quad e_{-\alpha_{i}} \mapsto f_{i}[0], \quad i=1, \ldots, N, \quad k_{\alpha_{0}}^{ \pm 1} \mapsto \prod_{i=1}^{N} k_{i}^{\mp 1} ; \\
e_{\alpha_{0}} \mapsto\left[f_{N}[0],\left[f_{N-1}[0], \ldots,\left[f_{2}[0], f_{1}[1]\right]_{q} \cdots\right]_{q}\right]_{q}, \\
e_{-\alpha_{0}} \mapsto\left[e_{N}[0],\left[e_{N-1}[0], \ldots,\left[e_{2}[0], e_{1}[-1]\right]_{q^{-1}} \ldots\right]_{q^{-1}}\right]_{q^{-1}} \\
{[B, A]_{q}=A B-q B A . }
\end{aligned}
$$

В последующем мы будем использовать естественное расширение $\bar{U}_{q}\left(\widehat{\mathfrak{s l}}_{N+1}\right)$ квантовой аффинной алгебры $U_{q}\left(\widehat{\mathfrak{s l}}_{N+1}\right)$, связанное с категорией представлений старшего веса (см., например, [13]). Это расширение может быть описано как пополнение алгебры $U_{q}\left(\widehat{\mathfrak{s l}}_{N+1}\right)$ в минимальной топологии, в которой действие элементов алгебры $U_{q}\left(\widehat{\mathfrak{s l}}_{N+1}\right)$ в любом представлении старшего веса непрерывно. Это пополнение является топологической алгеброй, действующей в категории представлений старшего веса. Выбор конкретного пополнения необходим для придания точного смысла сложным токам, которые будут определены ниже. Изменение пополнения изменит определение и аналитические свойства произведений этих токов.

2.1. Стандартные и токовые борелевские подалгебры в алгебре $U_{q}\left(\widehat{\mathfrak{s l}}_{N+1}\right)$. Обозначим через $U_{q}\left(\mathfrak{b}_{+}\right)$подалгебру в $U_{q}\left(\widehat{\mathfrak{s l}}_{N+1}\right)$, образованную элементами $e_{\alpha_{i}}$ и $k_{\alpha_{i}}^{ \pm 1}, i=0,1, \ldots, N$, в стандартной реализации алгебры $U_{q}\left(\widehat{\mathfrak{s l}}_{N+1}\right)$ при помощи образующих Шевалле. Обозначим через $U_{q}\left(\mathfrak{b}_{-}\right)$подалгебру в $U_{q}(\widehat{\mathfrak{g}})$, образованную элементами $e_{-\alpha_{i}}$ и $k_{\alpha_{i}}^{ \pm 1}$. Подалгебры $U_{q}\left(\mathfrak{b}_{ \pm}\right)$являются подалгебрами Хопфа в $U_{q}\left(\widehat{\mathfrak{s l}}_{N+1}\right)$ относительно стандартного коумножения $\Delta$. Они представляют собой $q$-деформации обертывающих алгебр стандартных борелевских подалгебр в алгебре Ли $\widehat{\mathfrak{s l}}_{N+1}$. Мы назовем их стандартными борелевскими подалгебрами. Они содержат подалгебры $U_{q}\left(\mathfrak{n}_{ \pm}\right)$, которые образованы элементами $e_{ \pm \alpha_{i}}, i=0,1, \ldots, N$. Подалгебра $U_{q}\left(\mathfrak{n}_{+}\right)$ является левым коидеалом в $U_{q}\left(\mathfrak{b}_{+}\right)$относительно стандартного коумножения, подалгебра $U_{q}\left(\mathfrak{n}_{-}\right)$- правый коидеал в $U_{q}\left(\mathfrak{b}_{-}\right)$относительно того же коумножения, т.е.

$$
\Delta\left(U_{q}\left(\mathfrak{n}_{+}\right)\right) \subset U_{q}\left(\mathfrak{b}_{+}\right) \otimes U_{q}\left(\mathfrak{n}_{+}\right), \quad \Delta\left(U_{q}\left(\mathfrak{n}_{-}\right)\right) \subset U_{q}\left(\mathfrak{n}_{-}\right) \otimes U_{q}\left(\mathfrak{b}_{-}\right) .
$$

Обозначим через $U_{F}$ подалгебру в $U_{q}\left(\widehat{\mathfrak{s l}}_{N+1}\right)$, образованную элементами $k_{i}^{ \pm 1}, f_{i}[n]$ $(n \in \mathbb{Z})$ и $h_{i}[n](n>0)$, и через $U_{E}-$ подалгебру в $U_{q}\left(\widehat{\mathfrak{s l}}_{N+1}\right)$, образованную элементами $k_{i}^{ \pm 1}, e_{i}[n](n \in \mathbb{Z})$ и $h_{i}[n](n<0), i=1, \ldots, N$. Они являются подалгебрами Хопфа в алгебре $U_{q}\left(\widehat{\mathfrak{s l}}_{N+1}\right)$ относительно коумножения $\Delta^{(\mathrm{D})}$. Мы будем называть их токовыми борелевскими подалгебрами. Токовые борелевские подалгебры $U_{F}$ и $U_{E}$ содержат подалгебры $U_{f}$ и $U_{e}$, образованные модами $f_{i}[n]$ и $e_{i}[n]$, соответственно $(n \in \mathbb{Z})$. Подалгебра $U_{f}$ является правым коидеалом в $U_{F}$ относительно $\Delta^{(\mathrm{D})}$, а подалгебра $U_{e}-$ левым коидеалом в $U_{E}$ относительно $\Delta^{(\mathrm{D})}$ :

$$
\Delta^{(\mathrm{D})}\left(U_{f}\right) \subset U_{f} \otimes U_{F}, \quad \Delta^{(\mathrm{D})}\left(U_{e}\right) \subset U_{E} \otimes U_{e}
$$


В последующем мы будем работать только с токовой борелевской подалгеброй $U_{F}$. Обозначим через $U_{F}^{+}$и $U_{f}^{-}$следующие пересечения стандартных и токовых борелевских подалгебр:

$$
U_{f}^{-}=U_{F} \cap U_{q}\left(\mathfrak{b}_{-}\right)=U_{f} \cap U_{q}\left(\mathfrak{n}_{-}\right), \quad U_{F}^{+}=U_{F} \cap U_{q}\left(\mathfrak{b}_{+}\right) .
$$

Эти пересечения удовлетворяют свойствам коидеальности [5]

$$
\Delta^{(\mathrm{D})}\left(U_{F}^{+}\right) \subset U_{F} \otimes U_{F}^{+}, \quad \Delta^{(\mathrm{D})}\left(U_{f}^{-}\right) \subset U_{f}^{-} \otimes U_{F},
$$

и умножение $m$ в $U_{q}\left(\widehat{\mathfrak{s l}}_{N+1}\right)$ индуцирует изоморфизм векторных пространств

$$
m: U_{f}^{-} \otimes U_{F}^{+} \rightarrow U_{F} .
$$

Определим проекционные операторы $P^{+}: U_{F} \rightarrow U_{F}^{+}$и $P^{-}: U_{F} \rightarrow U_{f}^{-}$следующими правилами:

$$
P^{+}\left(f_{-} f_{+}\right)=\varepsilon\left(f_{-}\right) f_{+}, \quad P^{-}\left(f_{-} f_{+}\right)=f_{-} \varepsilon\left(f_{+}\right),
$$

где $\varepsilon: U_{F} \rightarrow \mathbb{C}$ есть отображение коединицы и $f_{-} \in U_{f}^{-}, f_{+} \in U_{F}^{+}[5],[8],[9]$.

Основной целью настоящей работы является вычисление проекции $P^{+}$от произведения токов Дринфельда в алгебре $U_{q}\left(\widehat{\mathfrak{s l}}_{N+1}\right)$. Для решения этой задачи нам необходимо ввести сложные токи.

2.2. Сложные токи. В токовой реализации алгебры $U_{q}\left(\widehat{\mathfrak{s l}}_{N+1}\right)$ токи нумеруются простыми положительными корнями алгебры $\mathfrak{s l}_{N+1}$. Пусть $\Pi=\left\{\alpha_{1}, \ldots, \alpha_{N}\right\}-$ система простых положительных корней алгебры Ли $\mathfrak{s l}_{N+1}$. Для $1 \leqslant i<j \leqslant N+1$ обозначим $\Pi_{i j} \subset \Pi$ подсистему корней $\left\{\alpha_{i}, \ldots, \alpha_{j-1}\right\}$. Таким образом, $\Pi_{i, i+1}$ содержит один простой корень $\alpha_{i}$. Ток $f_{i}(z)$, заданный в $(2.4)$, отвечает корню $\alpha_{i}$ или подсистеме $\Pi_{i, i+1}$. Удобно обозначить этот ток как $f_{i, i+1}(z)$. Для подсистемы корней $\Pi_{i, j}, i<j-1$, определим сложный корень $\alpha[i, j]$ :

$$
\alpha[i, j]=\alpha_{i}+\alpha_{i+1}+\cdots+\alpha_{j-1} .
$$

Фиксируем следующее упорядочение среди всех положительных корней конечномерной алгебры $\mathfrak{s l}_{N+1}$ :

$$
\begin{aligned}
\alpha[1,2] & <\cdots<\alpha[1, N+1]<\alpha[2,3]<\cdots<\alpha[2, N+1]< \\
& <\cdots<\alpha[N-1, N]<\alpha[N-1, N+1]<\alpha[N, N+1] .
\end{aligned}
$$

Другими словами, будем говорить, что $\alpha[s, i]<\alpha[p, j]$, если $s<p$ или если $i<j$ при $s=p$.

Нашей задачей является определение токов $f_{i, j}(z) \in \bar{U}_{q}\left(\widehat{\mathfrak{s l}}_{N+1}\right)\left[\left[z, z^{-1}\right]\right]$, соответствующих сложным корням $\alpha[i, j]$. Эти токи (при $i<j-1$ ) определяются следующими рекуррентными соотношениями (для всех $s$ таких, что $i \leqslant s<j-1$ ):

$$
\begin{aligned}
f_{i, j}(z) & =-\operatorname{res}_{w=z q^{i-s-1}} f_{i, s+1}(z) f_{s+1, j}(w) \frac{d w}{w}= \\
& =\oint_{C_{0}} f_{i, s+1}(z) f_{s+1, j}(w) \frac{d w}{w}-\oint_{C_{\infty}} \frac{q^{-1} w-q^{i-s} z}{w-q^{i-s-1} z} f_{s+1, j}(w) f_{i, s+1}(z) \frac{d w}{w}
\end{aligned}
$$


или

$$
\begin{aligned}
f_{i, j}(z)= & \operatorname{res}_{w=z} f_{i, s+1}(w) f_{s+1, j}\left(q^{i-s-1} z\right) \frac{d w}{w}= \\
= & \oint_{C_{\infty}} f_{i, s+1}(w) f_{s+1, j}\left(q^{i-s-1} z\right) \frac{d w}{w}- \\
& \quad-\oint_{C_{0}} \frac{q^{-1} z-q w}{z-w} f_{s+1, j}\left(q^{i-s-1} z\right) f_{i, s+1}(w) \frac{d w}{w},
\end{aligned}
$$

где $C_{0}$ и $C_{\infty}$ являются контурами вокруг точек 0 и $\infty$, соответственно.

Коммутационные соотношения (2.5) позволяют получить выражения для сложных токов в виде произведения сдвинутых токов, отвечающих простым корням [8]:

$$
f_{i, j}(z)=\left(q-q^{-1}\right)^{j-i-1} f_{j-1}\left(q^{i-j+1} z\right) f_{j-2}\left(q^{i-j+2} z\right) \ldots f_{i+1}\left(q^{-1} z\right) f_{i}(z)
$$

для $1 \leqslant i<j \leqslant N+1$. Это доказывает, что формулы (2.14) и (2.15) дают один и тот же ответ для всех значений $s$.

Можно также вычислить формальные интегралы в (2.14):

$$
\begin{aligned}
f_{i, j}(z)=f_{i, s+1}(z) f_{s+1, j}[0]-q f_{s+1, j}[0] f_{i, s+1}(z)- \\
\quad-\left(q^{-1}-q\right) \sum_{k \geqslant 0} f_{s+1, j}[-k] f_{i, s+1}(z)\left(q^{i-s-1} z\right)^{k},
\end{aligned}
$$

где $f_{s+1, j}[n]-n$-я мода сложного тока $f_{s+1, j}(z)$. Это соотношение может быть использовано для вычисления проекции сложных токов. Действительно, рассмотрим его при $s=j-1$ и применим проекцию $P^{+}$к обеим сторонам равенства $(2.17)$. В соответствии с определениями (2.10) и (2.11) полубесконечная сумма в правой части соотношения (2.17) исчезает:

$$
\begin{aligned}
P^{+}\left(f_{i, j}(t)\right) & =S_{f_{j-1, j}[0]}\left(P^{+}\left(f_{i, j-1}(t)\right)\right) \equiv \\
& \equiv P^{+}\left(f_{i, j-1}(t)\right) \cdot f_{j-1, j}[0]-q f_{j-1, j}[0] \cdot P^{+}\left(f_{i, j-1}(t)\right) .
\end{aligned}
$$

Определим экранирующие операторь

$$
S_{f_{m, m+1}[0]}(x)=x \cdot f_{m, m+1}[0]-f_{m, m+1}[0] \cdot k_{m} x k_{m}^{-1},
$$

где $f_{k, k+1}[0]=f_{k}[0]=e_{-\alpha_{k}}$ является нулевой модой тока $f_{k, k+1}(t)$ (см. формулу $(2.9))$, а $k_{m}$ - нулевой модой картановского тока $\psi_{m}^{+}(z)$. Чтобы получить $(2.18)$ из (2.17), мы использовали свойство коммутативности экранирующих операторов с проекциями, которое сформулировано в лемме (см. приложение) и доказано в работе [9]. Применяя соотношение (2.18) несколько раз, получим

$$
P^{+}\left(f_{i, j}(t)\right)=S_{f_{j-1, j}[0]} \cdot S_{f_{j-2, j-1}[0]} \ldots S_{f_{i+1, i+2}[0]}\left(P^{+}\left(f_{i, i+1}(t)\right)\right),
$$

где $P^{+}\left(f_{i, i+1}(t)\right)=\sum_{k>0} f_{i, i+1}[k] t^{-k}$. 


\section{3. ОСНОВНОЙ РЕЗУЛЬТАТ}

Выберем пару $(i, j)$ положительных целых чисел такую, что $1 \leqslant i \leqslant j \leqslant N$. Паре $(i, j)$ сопоставим набор положительных целых чисел

$$
\bar{m}=\left\{m_{i}, \ldots, m_{j}\right\} \in \mathbb{Z}_{>0}^{j-i+1} .
$$

Пусть $\bar{m}$ и $\bar{n}$ - два таких набора, причем их элементы удовлетворяют неравенствам

$$
m_{a} \leqslant n_{a}, \quad a=i, \ldots, j
$$

Обозначим через $\bar{t}_{\bar{m}, \bar{n}}$ набор формальных переменных,

$$
\bar{t}_{\bar{m}, \bar{n}}=\left\{t_{m_{i}}^{i}, \ldots, t_{n_{i}}^{i} ; t_{m_{i+1}}^{i+1}, \ldots, t_{n_{i+1}}^{i+1} ; \ldots ; t_{m_{j}}^{j}, \ldots, t_{n_{j}}^{j}\right\}
$$

где верхний индекс $l$ в $t_{s_{l}}^{l}$ ассоциирован с простым корнем $\alpha_{l}$, а набор (3.3) - с подсистемой простых корней $\Pi_{i, j+1}$.

В дальнейшем будем использовать упрощенное обозначение для набора переменных: $\bar{t}_{\overline{1}, \bar{n}} \equiv \bar{t}_{\bar{n}}$, где $\overline{1}=\{1, \ldots, 1\}$, т.е.

$$
\bar{t}_{\bar{n}}=\left\{t_{1}^{i}, \ldots, t_{n_{i}}^{i} ; t_{1}^{i+1}, \ldots, t_{n_{i+1}}^{i+1} ; \ldots ; t_{1}^{j}, \ldots, t_{n_{j}}^{j}\right\} .
$$

Нашей целью является вычисление проекции

$$
\mathcal{W}^{1, N}\left(\bar{t}_{\bar{n}}\right)=P^{+}\left(f_{1,2}\left(t_{1}^{1}\right) \ldots f_{1,2}\left(t_{n_{1}}^{1}\right) \ldots f_{N, N+1}\left(t_{1}^{N}\right) \ldots f_{N, N+1}\left(t_{n_{N}}^{N}\right)\right)
$$

где $i=1, j=N$ в наборе $\bar{t}_{\bar{n}}$ :

$$
\bar{t}_{\bar{n}}=\left\{t_{1}^{1}, \ldots, t_{n_{1}}^{1} ; t_{1}^{2}, \ldots, t_{n_{2}}^{2} ; \ldots ; t_{1}^{N}, \ldots, t_{n_{N}}^{N}\right\}
$$

Пусть

$$
U_{F}^{+}\left(\bar{t}_{\bar{n}}\right)=U_{F}^{+}\left[\left(t_{1}^{1}\right)^{ \pm 1}, \ldots,\left(t_{n_{N}}^{N}\right)^{ \pm 1}\right]\left[\left[\frac{t_{2}^{1}}{t_{1}^{1}}, \ldots, \frac{t_{n_{1}}^{1}}{t_{n_{1}-1}^{1}}, \frac{t_{1}^{2}}{t_{n_{1}}^{1}}, \ldots, \frac{t_{n_{N}}^{N}}{t_{n_{N}-1}^{N}}, \frac{1}{t_{n_{N}}^{N}}\right]\right]
$$

- пространство формальных степенных рядов от переменных $t_{2}^{1} / t_{1}^{1}, t_{2}^{1} / t_{3}^{1}, \ldots$ $\ldots, t_{n_{N}}^{N} / t_{n_{N}-1}^{N}, 1 / t_{n_{N}}^{N}$, коэффициенты которых есть полиномы $U_{F}^{+}\left[\left(t_{1}^{1}\right)^{ \pm 1}, \ldots,\left(t_{n_{N}}^{N}\right)^{ \pm 1}\right]$, принимающие значения в подалгебре $U_{F}^{+}$.

Следуя результатам работ [5], [9], [10], мы будем называть элемент $\mathcal{W}^{1, N}\left(\bar{t}_{\bar{n}}\right)$ универсальной весовой функиией. Для формулировки основного результата нам необходимо определить понятия $q$-симметризации формальных рядов, упорядоченного разложения произведений токов, а также определить проекции произведений сложных токов. Это будет сделано ниже. 
3.1. q-Симметризация. Зафиксируем два набора положительных целых чисел $\bar{m}$ и $\bar{n}$, удовлетворяющих неравенствам (3.2). Пусть $S_{\bar{m}, \bar{n}}=S_{n_{i}-m_{i}+1} \times \cdots \times S_{n_{j}-m_{j}+1}$ - прямое произведение симметрических групп. Если $m_{s}=n_{s}$ для некоторого $s$, тогда набор переменных $\bar{t}_{\bar{m}, \bar{n}}$ содержит только одну переменную типа $s$. Соответствующая этому типу симметрическая группа является тривиальной. Группа $S_{\bar{m}, \bar{n}}$ естественным образом действует на функции от переменных $\bar{t}_{\bar{m}, \bar{n}}$, переставляя переменные одного типа. Для $\sigma=\sigma^{i} \times \cdots \times \sigma^{j} \in S_{\bar{m}, \bar{n}}$ положим

$$
{ }^{\sigma} \bar{t}_{\bar{n}, \bar{m}}=\left\{t_{\sigma^{i}\left(m_{i}\right)}^{i}, \ldots, t_{\sigma^{i}\left(n_{i}\right)}^{i} ; \ldots ; t_{\sigma^{j}\left(m_{j}\right)}^{j}, \ldots, t_{\sigma^{j}\left(n_{j}\right)}^{j}\right\} .
$$

Для произвольного формального ряда $F\left(\bar{t}_{\bar{m}, \bar{n}}\right) \in U_{F}^{+}\left(\bar{t}_{\bar{n}}\right) q$-симметризация означает, что

$$
\overline{\operatorname{Sym}}_{\bar{t}_{\bar{m}, \bar{n}}}\left(F\left(\bar{t}_{\bar{m}, \bar{n}}\right)\right)=\sum_{\sigma \in S_{\bar{m}, \bar{n}}} \prod_{i \leqslant s \leqslant j} \prod_{\substack{\ell<\ell^{\prime} \\ \sigma^{s}(\ell)>\sigma^{s}\left(\ell^{\prime}\right)}} \frac{q^{-1}-q t_{\ell^{\prime}}^{s} / t_{\ell}^{s}}{q-q^{-1} t_{\ell^{\prime}}^{s} / t_{\ell}^{s}} F\left({ }^{\sigma} \bar{t}_{\bar{m}, \bar{n}}\right) .
$$

Будем называть формальный ряд $F\left(\bar{t}_{\bar{m}, \bar{n}}\right) q$-симметричным, если

$$
\overline{\operatorname{Sym}}_{\bar{t}_{\bar{m}, \bar{n}}}\left(F\left(\bar{t}_{\bar{m}, \bar{n}}\right)\right)=\prod_{s=i}^{j}\left(n_{s}-m_{s}\right) ! F\left(\bar{t}_{\bar{m}, \bar{n}}\right) .
$$

Можно легко убедится в том, что $q$-симметризация произвольного ряда из пространства $U_{F}^{+}\left(\bar{t}_{\bar{n}}\right)$ является $q$-симметричным рядом.

3.2. Упорядоченное разложение произведений токов. Зафиксируем снова пару $(i, j)$ целых чисел таких, что $1 \leqslant i \leqslant j \leqslant N$, и сопоставим этой паре два набора $\bar{m}$ и $\bar{n}$, элементы которых удовлетворяют неравенствам (3.2). Пусть $\bar{s}-$ третий набор неотрицательных целых чисел (3.1), ассоциированный с парой $(i, j)$, причем $m_{a}-1 \leqslant s_{a} \leqslant n_{a}, a=i, \ldots, j$. Определим формальный ряд

$$
Z_{\bar{s}}^{i, j}\left(\bar{t}_{\bar{m}, \bar{n}}\right)=\prod_{\substack{s_{a}+1 \leqslant \ell \leqslant n_{a} \\ m_{a+1} \leqslant \ell^{\prime} \leqslant s_{a+1}}} \frac{q-t_{\ell^{\prime}}^{a+1} / t_{\ell}^{a}}{1-q t_{\ell^{\prime}}^{a+1} / t_{\ell}^{a}} .
$$

Заметим, что он не зависит от переменных $t_{m_{i}}^{i}, \ldots, t_{s_{i}}^{i}$ и $t_{s_{j}+1}^{j}, \ldots, t_{n_{j}}^{j}$. В частности, при $i=j$ этот ряд равен константе: $Z_{\bar{s}}^{i, i}\left(\bar{t}_{\bar{m}, \bar{n}}\right)=1$.

Для набора переменных $\bar{t}_{\bar{m}, \bar{n}}$ рассмотрим упорядоченное произведение

$$
\prod_{i \leqslant a \leqslant j}\left(\prod_{m_{a} \leqslant \ell \leqslant n_{a}} f_{a, a+1}\left(t_{\ell}^{a}\right)\right)
$$

где токи $f_{a, a+1}(t) \equiv f_{a}(t)$ определены в (2.4). Символ $\vec{\prod}_{a} A_{a}$ означает упорядоченное произведение некоммутирующих сомножителей $A_{a}$ такое, что $A_{i}$ находится слева от $A_{j}$, если $i<j$. Используя свойства проекционных операторов [7], [8], мы можем записать упорядоченное произведение токов, отвечающих простым корням и зависящих от набора переменных (3.3), в нормально упорядоченном виде. Под нормально 
упорядоченным (или просто упорядоченным) представлением элемента $F \in U_{F}$ мы будем понимать его представление в виде суммы произведений $F=\sum_{i} F_{-}^{(i)} F_{+}^{(i)}$, где $F_{-}^{(i)} \in U_{f}^{-}$и $F_{+}^{(i)} \in U_{F}^{+}$. Сформулируем это утверждение как следующее

ПРЕДЛОЖЕНИЕ 1. Имеет место равенство

$$
\begin{aligned}
\prod_{i \leqslant a \leqslant j}( & \left.\prod_{m_{a} \leqslant \ell \leqslant n_{a}} f_{a, a+1}\left(t_{\ell}^{a}\right)\right)=\sum_{m_{a}-1 \leqslant s_{a} \leqslant n_{a}} \prod_{i \leqslant a \leqslant j} \frac{1}{\left(\prod_{a}-m_{a}+1\right) !\left(n_{a}-s_{a}\right) !} \times \\
& \times \overline{\operatorname{Sym}}_{\bar{t}_{\bar{m}, \bar{n}}}\left(Z_{\bar{s}}^{i, j}\left(\bar{t}_{\bar{m}, \bar{n})} P^{-}\left(\prod_{i \leqslant a \leqslant j} f_{m_{a} \leqslant \ell \leqslant s_{a}}\left(t_{\ell, a+1}^{a}\right)\right)\right) \times\right. \\
& \left.\times P^{+}\left(\prod_{i \leqslant a \leqslant j}\left(\prod_{s_{a}+1 \leqslant \ell \leqslant n_{a}} f_{a, a+1}\left(t_{\ell}^{a}\right)\right)\right)\right) .
\end{aligned}
$$

3.3. Определение струн. Свяжем с парой целых чисел $(i, j)$ таких, что $1 \leqslant$ $i \leqslant j \leqslant N$, набор $\bar{k}=\left\{k_{i}, \ldots, k_{j}\right\}$ неотрицательных целых чисел, удовлетворяющих условию неубывания $k_{i} \geqslant k_{i+1} \geqslant \cdots \geqslant k_{j} \geqslant 0$. Для набора $\bar{k}$ рассмотрим совокупность переменных $\bar{t}_{\bar{k}}$ (3.4). Для этой совокупности определим упорядоченное произведение токов

$$
F_{k_{i}, \ldots, k_{j}}\left(\bar{t}_{\bar{k}}\right)=\prod_{i \leqslant s \leqslant j}\left(\prod_{k_{i}-k_{s}+1 \leqslant \ell \leqslant k_{i}-k_{s}+1} f_{i, s+1}\left(t_{\ell}^{i}\right)\right)
$$

которое зависит только от переменных типа $i\left(t_{1}^{i}, \ldots, t_{k_{i}}^{i}\right)$ и положим $k_{j+1}=0$. Будем называть такое произведение токов струной, соответствующей набору $\bar{k}$ и подсистеме корней $\Pi_{i, j+1}$.

Для набора переменных $\bar{t}_{\bar{k}}$ определим элемент $W^{i, j}\left(\bar{t}_{\bar{k}}\right) \in U_{F}^{+}$равенством

$$
W^{i, j}\left(\bar{t}_{\bar{k}}\right)=P^{+}\left(F_{k_{i}, \ldots, k_{j}}\left(\bar{t}_{\bar{k}}\right)\right) \prod_{a=i}^{j-1} \prod_{\ell=1}^{k_{a+1}} \frac{1}{1-q t_{\ell}^{a+1} / t_{k_{a}-k_{a+1}+\ell}^{a}} \prod_{\ell^{\prime}=1}^{\ell-1} \frac{q-t_{\ell^{\prime}}^{a+1} / t_{k_{a}-k_{a+1}+\ell}^{a}}{1-q t_{\ell^{\prime}}^{a+1} / t_{k_{a}-k_{a+1}+\ell}^{a}} .
$$

При $i=j$ струна (3.11) является упорядоченным произведением токов, соответствующих одному корню $\alpha_{i}$ :

$$
F_{k_{i}}\left(\bar{t}_{\bar{k}}\right)=f_{i, i+1}\left(t_{1}^{i}\right) f_{i, i+1}\left(t_{2}^{i}\right) \ldots f_{i, i+1}\left(t_{k_{i}-1}^{i}\right) f_{i, i+1}\left(t_{k_{i}}^{i}\right)
$$

В этом случае $W^{i, i}\left(\bar{t}_{\bar{k}}\right)$ означает проекцию этого произведения:

$$
W^{i, i}\left(\bar{t}_{\bar{k}}\right)=P^{+}\left(f_{i, i+1}\left(t_{1}^{i}\right) f_{i, i+1}\left(t_{2}^{i}\right) \ldots f_{i, i+1}\left(t_{k_{i}-1}^{i}\right) f_{i, i+1}\left(t_{k_{i}}^{i}\right)\right) .
$$

3.4. Универсальная весовая функция как проекция. Пусть набор $\bar{n}$ тот же, что и в (3.6). Пусть $\{\bar{k}\}$ - треугольная матрица с неотрицательными целыми 
элементами,

$$
\{\bar{k}\}=\left(\begin{array}{ccccc}
k_{1}^{1} & k_{2}^{1} & \ldots & k_{N-1}^{1} & k_{N}^{1} \\
& k_{2}^{2} & \ldots & k_{N-1}^{2} & k_{N}^{2} \\
& & \ldots & k_{N-1}^{3} & k_{N}^{3} \\
& & \ddots & \vdots & \vdots \\
& & & k_{N-1}^{N-1} & k_{N}^{N-1} \\
& & & & k_{N}^{N}
\end{array}\right) .
$$

Будем говорить, что матрица $\{\bar{k}\}$ является $\bar{n}$-допустимой, обозначая это как $\{\bar{k}\} \prec \bar{n}$, если ее элементы не возрастают вдоль строк, а суммы их по столбцам совпадают с элементами набора $\bar{n}$ :

$$
\begin{gathered}
k_{1}^{1} \geqslant k_{2}^{1} \geqslant \cdots \geqslant k_{N}^{1}, \quad k_{2}^{2} \geqslant k_{3}^{2} \geqslant \cdots \geqslant k_{N}^{2}, \quad \ldots, \quad k_{N-1}^{N-1} \geqslant k_{N}^{N-1}, \\
k_{1}^{1}=n_{1}, \quad k_{2}^{1}+k_{2}^{2}=n_{2}, \quad \ldots, \quad k_{N}^{1}+\cdots+k_{N}^{N}=n_{N} .
\end{gathered}
$$

Обозначим через $\bar{k}^{i}$ ненулевую часть $i$-й строки матрицы $\{\bar{k}\}, \bar{k}^{i}=\left\{k_{i}^{i}, k_{i+1}^{i}, \ldots, k_{N}^{i}\right\}$.

Для любого $i=2, \ldots, N$ и допустимой матрицы $\{\bar{k}\}$ введем три набора целых чисел

$$
\begin{aligned}
\bar{m}^{i}(\{\bar{k}\}) & =\left\{k_{i}^{1}+\cdots+k_{i}^{i-2}+1, \ldots, k_{N}^{1}+\cdots+k_{N}^{i-2}+1\right\}, \\
\bar{l}^{i}(\{\bar{k}\}) & =\left\{k_{i}^{1}+\cdots+k_{i}^{i-1}, \ldots, k_{N}^{1}+\cdots+k_{N}^{i-1}\right\}, \\
\bar{n}^{i} & =\left\{n_{i}, \ldots, n_{N}\right\} .
\end{aligned}
$$

Пусть $\bar{m}^{2}(\{\bar{k}\})=\{1, \ldots, 1\}$ и $\bar{l}^{1}(\{\bar{k}\})=\{0, \ldots, 0\}$. Используя эти наборы целых чисел, введем два набора переменных, ассоциированных с парой $(i, N)$ или с подсистемой корней $\Pi_{i, N+1}$,

$$
\bar{t}_{\bar{m}^{i}(\{\bar{k}\}), \bar{n}^{i}}, \quad \hat{t}_{\bar{l}^{i}(\{\bar{k}\}), \bar{k}^{i}} \equiv \bar{t}_{\bar{l}^{i}(\{\bar{k}\})+\overline{1}, \bar{l}^{i}(\{\bar{k}\})+\bar{k}^{i},}
$$

где первый набор определен формулой (3.3), а второй есть

$$
\hat{t}_{\bar{l}^{i}(\{\bar{k}\}), \bar{k}^{i}}=\left\{t_{l_{i}^{i}+1}^{i}, \ldots, t_{l_{i}^{i}+k_{i}^{i}}^{i}, \quad t_{l_{i+1}^{i}+1}^{i+1}, \ldots, t_{l_{i+1}^{i}+k_{i+1}^{i}}^{i+1}, \quad \ldots, \quad t_{l_{N}^{i}+1}^{N}, \ldots, t_{l_{N}^{i}+k_{N}^{i}}^{N}\right\} .
$$

Сформулируем основной результат этой работы как следующую теорему.

Теорема 1. Универсальная весовая функиия (3.5) может быть представлена как полная q-симметризация по всем группам переменных от суммы по всем $\bar{n}$ допустимым матрицам $\{\bar{k}\}$ от упорядоченного произведения проекиий струн с рациональными коэфбициентами:

$$
\begin{aligned}
\mathcal{W}^{1, N}\left(\bar{t}_{\bar{n}}\right)=\overline{\operatorname{Sym}}_{\bar{t}_{\bar{n}}} \sum_{\{\bar{k}\} \prec \bar{n}}\left(\prod_{l=1}^{N} \prod_{m=l}^{N} \frac{1}{\left(k_{m}^{l}-k_{m+1}^{l}\right) !} \times\right. \\
\quad \times \prod_{i=2}^{N} Z_{\bar{l}^{i}(\{\bar{k}\})}^{i, N}\left(\bar{t}_{\left.\bar{m}^{i}(\{\bar{k}\}), \bar{n}^{i}\right)} \prod_{1 \leqslant i \leqslant N} W^{i, N}\left(\hat{t}_{\left.\bar{l}^{i}(\{\bar{k}\}), \bar{k}^{i}\right)}\right)\right.
\end{aligned}
$$

(в данном равенстве $k_{N+1}^{l}=0$ ). 
3.5. Пример весовой функции. В этом разделе мы выпишем в явном виде все составляющие формулы (3.16) в случае квантовой аффинной алгебры $U_{q}\left(\widehat{\mathfrak{s l}}_{4}\right)$, $N=3$. Чтобы уменьшить количество индексов, используемых при обозначении переменных $\bar{t}_{\bar{n}}$, переобозначим их следующим образом:

$$
\left\{t_{1}^{1}, \ldots, t_{n_{1}}^{1} ; t_{2}^{2}, \ldots, t_{n_{2}}^{2} ; t_{1}^{3}, \ldots, t_{n_{3}}^{3}\right\} \rightarrow\left\{t_{1}, \ldots, t_{a} ; v_{1}, \ldots, v_{b} ; u_{1}, \ldots, u_{c}\right\}
$$

для любых положительных целых $a, b, c$. Переобозначим также матричные элементы допустимой матрицы $\{\bar{k}\}$ :

$$
\{\bar{k}\}=\left(\begin{array}{ccc}
k_{1}^{1} & k_{2}^{1} & k_{3}^{1} \\
0 & k_{2}^{2} & k_{3}^{2} \\
0 & 0 & k_{3}^{3}
\end{array}\right) \rightarrow\left(\begin{array}{ccc}
a & \kappa_{1} & \kappa_{2} \\
0 & b-\kappa_{1} & \kappa_{3} \\
0 & 0 & c-\kappa_{2}-\kappa_{3}
\end{array}\right),
$$

тогда суммирование в выражении для универсальной весовой функции будет вестись по неотрицательным целым числам $\kappa_{1}, \kappa_{2}$ и $\kappa_{3}$, удовлетворяющим условиям допустимости

$$
a \geqslant \kappa_{1} \geqslant \kappa_{2} \geqslant 0, \quad b-\kappa_{1} \geqslant \kappa_{3} \geqslant 0, \quad c-\kappa_{2}-\kappa_{3} \geqslant 0 .
$$

Наборы (3.14) будут иметь вид

$$
\begin{array}{llll} 
& \bar{l}^{1}=\{0,0,0\}, & \bar{k}^{1}=\left\{a, \kappa_{1}, \kappa_{2}\right\}, & \\
\bar{m}^{2}=\{1,1\}, & \bar{l}^{2}=\left\{\kappa_{1}, \kappa_{2}\right\}, & \bar{k}^{2}=\left\{b-\kappa_{1}, \kappa_{3}\right\}, & \bar{n}^{2}=\{b, c\}, \\
\bar{m}^{3}=\left\{\kappa_{2}+1\right\}, & \bar{l}^{3}=\left\{\kappa_{2}+\kappa_{3}\right\}, & \bar{k}^{3}=\left\{c-\kappa_{2}-\kappa_{3}\right\}, & \bar{n}^{3}=\{c\} .
\end{array}
$$

Соответствующие наборы переменных суть

$$
\begin{aligned}
\bar{t}_{\bar{m}^{2}, \bar{n}^{2}} & =\left\{v_{1}, \ldots, v_{b} ; u_{1}, \ldots, u_{c}\right\}, \quad \hat{t}_{\bar{l}^{1}, \bar{k}^{1}}=\left\{t_{1}, \ldots, t_{a} ; v_{1}, \ldots, v_{\kappa_{1}} ; u_{1}, \ldots, u_{\kappa_{2}}\right\}, \\
\hat{t}_{\bar{l}^{1}, \bar{k}^{2}} & =\left\{v_{\kappa_{1}+1}, \ldots, v_{b} ; u_{\kappa_{2}+1}, \ldots, u_{\kappa_{2}+\kappa_{3}}\right\}, \quad \hat{t}_{\bar{l}^{3}, \bar{k}^{3}}=\left\{u_{\kappa_{2}+\kappa_{3}+1}, \ldots, u_{c}\right\} .
\end{aligned}
$$

С целью упрощения дальнейших формул для двух наборов переменных $\left\{u_{1}, \ldots, u_{k}\right\}$ и $\left\{v_{1}, \ldots, v_{k}\right\}$ введем следующий формальный ряд:

$$
Y\left(u_{1}, \ldots, u_{k} ; v_{1}, \ldots, v_{k}\right)=\prod_{i=1}^{k} \frac{1}{1-v_{i} / u_{i}} \prod_{j=1}^{i-1} \frac{q-q^{-1} v_{j} / u_{i}}{1-v_{j} / u_{i}} .
$$

Используя это обозначение, получим

$$
\begin{aligned}
& W^{1,3}\left(\hat{t}_{\bar{l}^{1}, \bar{k}^{1}}\right)=Y\left(q^{-1} v_{\kappa_{1}-\kappa_{2}+1}, \ldots, q^{-1} v_{\kappa_{1}} ; u_{1}, \ldots, u_{\kappa_{2}}\right) \times \\
& \quad \times Y\left(q^{-1} t_{a-\kappa_{1}+1}, \ldots, q^{-1} t_{a} ; v_{1}, \ldots, v_{\kappa_{1}}\right) \times \\
& \quad \times P^{+}\left(f_{1,2}\left(t_{1}\right) \ldots f_{1,2}\left(t_{a-\kappa_{1}}\right) f_{1,3}\left(t_{a-\kappa_{1}+1}\right) \ldots f_{1,3}\left(t_{a-\kappa_{2}}\right) f_{1,4}\left(t_{a-\kappa_{2}+1}\right) \ldots f_{1,4}\left(t_{a}\right)\right), \\
& \quad W^{2,3}\left(\hat{t}_{\bar{l}^{2}, \bar{k}^{2}}\right)=Y\left(q^{-1} v_{b-\kappa_{3}+1}, \ldots, q^{-1} v_{b} ; u_{\kappa_{2}+1}, \ldots, u_{\kappa_{2}+\kappa_{3}}\right) \times \\
& \quad \times P^{+}\left(f_{2,3}\left(v_{\kappa_{1}+1}\right) \ldots f_{2,3}\left(v_{b-\kappa_{3}}\right) f_{2,4}\left(v_{b-\kappa_{3}+1}\right) \ldots f_{2,4}\left(v_{b}\right)\right) \\
& W^{3,3}\left(\hat{t}_{\bar{l}^{3}, \bar{k}^{3}}\right)=P^{+}\left(f_{3,4}\left(u_{\kappa_{2}+\kappa_{3}+1}\right) \ldots f_{3,4}\left(u_{c}\right)\right)
\end{aligned}
$$




$$
Z_{\bar{l}^{2}}^{2,3}\left(\bar{t}_{\bar{m}^{2}, \bar{n}^{2}}\right)=\prod_{\substack{\kappa_{1}+1 \leqslant \ell \leqslant b \\ 1 \leqslant \ell^{\prime} \leqslant \kappa_{2}}} \frac{q-u_{\ell^{\prime}} / v_{\ell}}{1-q u_{\ell^{\prime}} / v_{\ell}} .
$$

3.6. Проекции струн. В этом пункте мы приведем выражение для проекции струны (3.11). Чтобы сделать это, введем для набора переменных $\left\{u_{1}, \ldots, u_{b}\right\}$ множество рациональных функций от переменной $u$ вида

$$
\begin{aligned}
\varphi_{u_{m}}\left(u ; u_{1}, \ldots, u_{b}\right)= & \prod_{k<m}^{b} \frac{1-u / u_{k}}{1-u_{m} / u_{k}} \prod_{k>m}^{b} \frac{u / u_{m}-u_{k} / u_{m}}{1-u_{k} / u_{m}} \times \\
& \times \prod_{k=1}^{b} \frac{q-q^{-1} u_{m} / u_{k}}{q-q^{-1} u / u_{k}}, \quad m=1, \ldots, b .
\end{aligned}
$$

Для каждого тока $f_{i, j}(u)$ рассмотрим линейную комбинацию токов

$$
f_{i, j}\left(u ; u_{1}, \ldots, u_{b}\right)=f_{i, j}(u)-\sum_{m=1}^{b} \varphi_{u_{m}}\left(u ; u_{1}, \ldots, u_{b}\right) f_{i, j}\left(u_{m}\right) .
$$

Напомним, что проекция сложного тока определена через экранирующие операторы:

$$
P^{+}\left(f_{i, j}(u)\right)=S_{f_{j-1}[0]} \cdot S_{f_{j-2}[0]} \ldots S_{f_{i+1}[0]}\left(P^{+}\left(f_{i}(u)\right)\right) .
$$

Проекция линейной комбинации сложных токов (3.20) задается аналогичной формулой

$$
P^{+}\left(f_{i, j}\left(u ; u_{1}, \ldots, u_{b}\right)\right)=S_{f_{j-1}[0]} \cdot S_{f_{j-2}[0]} \ldots S_{f_{i+1}[0]}\left(P^{+}\left(f_{i}\left(u ; u_{1}, \ldots, u_{b}\right)\right)\right) .
$$

Проекция струны (3.11) задается следующим факторизованным выражением:

$$
\begin{aligned}
& P^{+}\left(F_{k_{i}, \ldots, k_{j}}\left(\bar{t}_{\bar{k}}\right)\right)=\prod_{i \leqslant s<s^{\prime} \leqslant j}\left(\prod_{\substack{k_{s+1} \leqslant \ell \leqslant k_{s}-1 \\
k_{s^{\prime}+1} \leqslant \ell^{\prime} \leqslant k_{s^{\prime}}-1}} \frac{q-q^{-1} t_{k_{i}-\ell^{\prime}}^{i} / t_{k_{i}-\ell}^{i}}{1-t_{k_{i}-\ell^{\prime}}^{i} / t_{k_{i}-\ell}^{i}}\right) \times \\
& \times \prod_{1 \leqslant \ell<\ell^{\prime} \leqslant k_{i}} \frac{q^{-1}-q t_{\ell^{\prime}}^{i} / t_{\ell}^{i}}{q-q^{-1} t_{\ell^{\prime}}^{i} / t_{\ell}^{i}} \prod_{i \leqslant s \leqslant j}\left(\prod_{k_{i}-k_{s}+1 \leqslant \ell \leqslant k_{i}-k_{s+1}} P^{+}\left(f_{i, s+1}\left(t_{\ell}^{i} ; t_{\ell+1}^{i}, \ldots, t_{k_{i}}^{i}\right)\right)\right)
\end{aligned}
$$

где $k_{j+1}=0$. Доказательство этой формулы аналогично доказательству, приведенному в работе [9], для проекций струн в алгебре $U_{q}\left(\widehat{\mathfrak{s l}}_{3}\right)$. Проекции токов могут быть связаны с гауссовым разложением $L$-операторов в алгебре $U_{q}\left(\widehat{\mathfrak{s l}}_{N+1}\right)$ (см. [10]).

\section{4. ДОКАЗАТЕЛЬСТВА ОСНОВНЫХ УТВЕРЖДЕНИЙ}

4.1. Доказательство упорядоченного разложения. Доказательство предложения 1 является чисто комбинаторным и основано на следующем свойстве проекций (2.11): для любого элемента $F \in U_{F}=U_{f}^{-} \cdot U_{F}^{+}$такого, что $\Delta^{(\mathrm{D})}(F)=\sum_{i} F_{i}^{\prime} \otimes F_{i}^{\prime \prime}$, справедливо равенство

$$
F=\sum_{i} P^{-}\left(F_{i}^{\prime \prime}\right) \cdot P^{+}\left(F_{i}^{\prime}\right)
$$


Применение этого свойства к упорядоченному произведению токов в левой части равенства (3.10) приводит к сумме слагаемых таких, что элементы $F_{i}^{\prime \prime}$ будут состоять из смешанных произведений полных и картановских токов. Для вычисления проекции $P^{-}$от этих слагаемых мы должны переставить все картановские токи направо, используя коммутационные соотношения из (2.6). При перестановке полных и картановских токов, отвечающих одному корню, появляются рациональные множители, которые затем включаются в $q$-симметризацию по переменным, отвечающим этому корню. Переставляя полные и картановские токи, отвечающие соседним корням, получим рациональные множители - функции $Z_{\bar{s}}^{i, j}\left(\bar{t}_{\bar{m}, \bar{n}}\right)$. Продемонстрируем, как работает этот механизм на простейшем нетривиальном примере.

Пусть $F=f_{1}\left(t_{1}\right) f_{1}\left(t_{2}\right) f_{2}(u)$. Это пример соответствует $i=1, j=2, m_{1}=1$, $n_{1}=2, m_{2}=1$ и $n_{2}=1$ в (3.10). Из (2.8) получим

$$
\begin{aligned}
\Delta^{(\mathrm{D})}\left(f_{1}(\right. & \left.\left.t_{1}\right) f_{1}\left(t_{2}\right) f_{2}(u)\right)=1 \otimes f_{1}\left(t_{1}\right) f_{1}\left(t_{2}\right) f_{2}(u)+ \\
& +f_{1}\left(t_{1}\right) \otimes f_{1}\left(t_{2}\right) f_{2}(u) \psi_{1}^{+}\left(t_{1}\right) \frac{q^{-1} t_{1}-q t_{2}}{q t_{1}-q^{-1} t_{2}} \frac{q t_{1}-u}{t_{1}-q u}+ \\
& +f_{1}\left(t_{2}\right) \otimes f_{1}\left(t_{1}\right) f_{2}(u) \psi_{1}^{+}\left(t_{2}\right) \frac{q t_{2}-u}{t_{2}-q u}+ \\
& +f_{2}(s) \otimes f_{1}\left(t_{1}\right) f_{1}\left(t_{2}\right) \psi_{2}^{+}(u)+ \\
& +f_{1}\left(t_{1}\right) f_{1}\left(t_{2}\right) \otimes f_{2}(u) \psi_{1}^{+}\left(t_{1}\right) \psi_{1}^{+}\left(t_{2}\right) \frac{q t_{1}-u}{t_{1}-q u} \frac{q t_{2}-u}{t_{2}-q u}+ \\
& +f_{1}\left(t_{1}\right) f_{2}(u) \otimes f_{1}\left(t_{2}\right) \psi_{1}^{+}\left(t_{1}\right) \psi_{2}^{+}(u) \frac{q^{-1} t_{1}-q t_{2}}{q t_{1}-q^{-1} t_{2}}+ \\
& +f_{1}\left(t_{2}\right) f_{2}(u) \otimes f_{1}\left(t_{1}\right) \psi_{1}^{+}\left(t_{2}\right) \psi_{2}^{+}(u)+ \\
& +f_{1}\left(t_{1}\right) f_{1}\left(t_{2}\right) f_{2}(u) \otimes \psi_{1}^{+}\left(t_{1}\right) \psi_{1}^{+}\left(t_{2}\right) \psi_{2}^{+}(u) .
\end{aligned}
$$

Применяя формулу разложения (4.1), найдем

$$
\begin{array}{rlrl}
f_{1}\left(t_{1}\right) f_{1}\left(t_{2}\right) f_{2}(u)=\overline{\operatorname{Sym}}_{t_{1}, t_{2}}\left[\frac{1}{2} P^{-}\left(f_{1}\left(t_{1}\right) f_{1}\left(t_{2}\right) f_{2}(u)\right)+\right. & \left(s_{1}, s_{2}=2,1\right) \\
& +P^{-}\left(f_{1}\left(t_{1}\right) f_{2}(u)\right) \cdot P^{+}\left(f_{1}\left(t_{2}\right)\right) \frac{q-u / t_{2}}{1-q u / t_{2}}+ & \left(s_{1}, s_{2}=1,1\right) \\
& +\frac{1}{2} P^{-}\left(f_{2}(u)\right) \cdot P^{+}\left(f_{1}\left(t_{1}\right) f_{1}\left(t_{2}\right)\right) \frac{q-u / t_{1}}{1-q u / t_{1}} \frac{q-u / t_{2}}{1-q u / t_{2}}+ & \left(s_{1}, s_{2}=0,1\right) \\
& +\frac{1}{2} P^{-}\left(f_{1}\left(t_{1}\right) f_{1}\left(t_{2}\right)\right) \cdot P^{+}\left(f_{2}(u)\right)+ & \left(s_{1}, s_{2}=2,0\right) \\
& +P^{-}\left(f_{1}\left(t_{1}\right)\right) \cdot P^{+}\left(f_{1}\left(t_{2}\right) f_{2}(u)\right)+ & \left(s_{1}, s_{2}=1,0\right) \\
+ & \left.\frac{1}{2} P^{+}\left(f_{1}\left(t_{1}\right) f_{1}\left(t_{2}\right) f_{2}(u)\right)\right] . & \left(s_{1}, s_{2}=0,0\right)
\end{array}
$$

Легко видеть, что последнее равенство есть частный случай (3.10); чтобы облегчить сравнение этих формул, в последнем равенстве мы выписали явно значения переменных суммирования $s_{1}$ и $s_{2}, 0 \leqslant s_{1} \leqslant 2,0 \leqslant s_{2} \leqslant 1$. 
4.2. Доказательство теоремы 1. Для доказательства теоремы 1 поступим следующим образом. Сначала представим произведение токов

$$
f_{2,3}\left(t_{1}^{2}\right) \ldots f_{2,3}\left(t_{n_{2}}^{2}\right) \ldots f_{N, N+1}\left(t_{1}^{N}\right) \ldots f_{N, N+1}\left(t_{n_{N}}^{N}\right)
$$

в упорядоченном виде, используя предложение 1. Заметим, что это разложение содержит $q$-симметризацию по переменным $t_{1}^{i}, \ldots, t_{n_{i}}^{i}, i=2, \ldots, N$.

Заменим суммирование по переменным $s_{i}, i=2, \ldots, N$, использованное в разложении (3.10), на суммирование по переменным $k_{i}^{1}$. Предложения 2 и 3 , сформулированные ниже, показывают, что эти переменные суммирования удовлетворяют ограничениям

$$
n_{1}=k_{1}^{1} \geqslant k_{2}^{1} \geqslant k_{3}^{1} \geqslant \cdots \geqslant k_{N}^{1},
$$

и таким образом появляется суммирование по первой строчке допустимой матрицы $\{\bar{k}\}$.

Следующим шагом является перемещение с учетом коммутационных соотношений отрицательной проекции от произведения токов

$$
P^{-}\left(\prod_{2 \leqslant i \leqslant N} \prod_{1 \leqslant \ell \leqslant k_{i}^{1}} f_{i}\left(t_{\ell}^{i}\right)\right)
$$

налево через произведение токов $f_{1}\left(t_{1}^{1}\right) \ldots f_{1}\left(t_{n_{1}}^{1}\right)$, отвечающих первому корню, с целью получить элемент $W^{1, N}$. Этот шаг представляет собой основную техническую сложность конструкции, и данное вычисление становится возможным благодаря специальному представлению элемента (4.3), принадлежащего $U_{f}^{-}$.

Пусть $U_{2}^{-}$и $U_{3, N}^{-}$- подалгебры в $U_{f}^{-}$, которые содержат моды отрицательных проекций токов $P^{-}\left(f_{2,3}(t)\right)$ и $P^{-}\left(f_{i, j}(t)\right)$ для $3 \leqslant i<j \leqslant N+1$, соответственно. Имеем следующее

ПреДЛОЖЕНИЕ 2. 1. Элемент (4.3) может быть представлен в виде

$$
\begin{aligned}
& \frac{1}{\left(k_{2}^{1}-k_{3}^{1}\right) ! \ldots\left(k_{N-1}^{1}-k_{N}^{1}\right) ! k_{N}^{1} !} \times \\
& \times \overline{\operatorname{Sym}}_{\bar{t}_{\bar{k} 1}^{2}, \ldots, \bar{t}_{\bar{k}^{1}}}^{k_{1}^{1}, \ldots, k_{N}^{1}}\left(\prod_{i=2}^{N-1} Y\left(q^{-1} \tilde{t}_{\bar{k}^{1}}^{i} ; \bar{t}_{\bar{k}^{1}}^{i+1}\right) P^{-}\left(\prod_{3 \leqslant i \leqslant N+1}^{\longrightarrow} \prod_{k_{2}^{1}-k_{i-1}^{1}}^{\longrightarrow} f_{2 \leqslant j \leqslant k_{2}^{1}-k_{i}^{1}} f_{2, i}\left(t_{j}^{2}\right)\right)\right)+ \\
& +\mathcal{F}_{3, N} \cdot \mathcal{F}_{2},
\end{aligned}
$$

где элементы $\mathcal{F}_{2} \in U_{2}^{-}$u $\mathcal{F}_{3, N} \in U_{3, N}^{-}$таковы, что $\varepsilon\left(\mathcal{F}_{3, N}\right)=0$.

2. Часть выражения (4.4), содержащая q-симметризачию, отлична от нуля, только если

$$
k_{2}^{1} \geqslant k_{3}^{1} \geqslant \cdots \geqslant k_{N-1}^{1} \geqslant k_{N}^{1} .
$$

Здесъ $\bar{t}_{\bar{k}}^{l} \subset \bar{t}_{\bar{k}}$ означает поднабор, состоящий из $k_{l}$ переменных $\left\{t_{1}^{l}, \ldots, t_{k_{l}}^{l}\right\}$, ассоциированных с корнем $\alpha_{l}$; набор $\tilde{t}_{\bar{k}}^{l} \subset \bar{t}_{\bar{k}}$ означает $k_{l+1}$ переменньх $\left\{t_{k_{l}-k_{l+1}+1}^{l}, \ldots, t_{k_{l}}^{l}\right\}$, взятых в естественном порядке, например, $Y\left(q^{-1} \tilde{t}_{\bar{k}}^{s} ; \bar{t}_{\bar{k}}^{s+1}\right)$ означает, что

$$
Y\left(q^{-1} \tilde{t}_{\bar{k}}^{s} ; \bar{t}_{\bar{k}}^{s+1}\right)=Y\left(q^{-1} t_{k_{s}-k_{s+1}+1}^{s}, \ldots, q^{-1} t_{k_{s}}^{s} ; t_{1}^{s+1}, \ldots, t_{k_{s+1}}^{s+1}\right) .
$$


ДОКАЗАТЕЛЬСТво этого и последующего предложений можно найти в приложении. В (4.4) следует положить $k_{N+1}^{1}=0$. В (4.4) в обозначении $q$-симметризации мы явно указали, что она ведется по $k_{a}^{1}$ переменным $\bar{t}_{\bar{k}^{1}}^{a}=\left\{t_{1}^{a}, \ldots, t_{k_{a}^{1}}^{a}\right\}, a=2, \ldots, N$.

Слагаемые вида $\mathcal{F}_{3, N} \cdot \mathcal{F}_{2}$ в $(4.4)$ не дают вклада в универсальную весовую функцию (3.5), так как

$$
P^{+}\left(\prod_{1 \leqslant \ell \leqslant n_{1}} f_{1,2}\left(t_{\ell}^{1}\right) \cdot \mathcal{F}_{3, N} \cdot \mathcal{F}_{2}\right)=P^{+}\left(\mathcal{F}_{3, N} \cdot \prod_{1 \leqslant \ell \leqslant n_{1}}^{\longrightarrow} f_{1,2}\left(t_{\ell}^{1}\right) \cdot \mathcal{F}_{2}\right)=0
$$

из-за коммутативности элементов из $U_{3, N}^{-}$с модами тока, отвечающими первому корню, и вследствие того, что проекция $P^{+}\left(\mathcal{F}_{3, N} \cdot \mathcal{F}\right)=0$ для любых элементов $\mathcal{F}_{3, N} \in U_{3, N}^{-}, \mathcal{F} \in U_{f}$ таких, что $\varepsilon\left(\mathcal{F}_{3, N}\right)=0$.

Из предложения 2 следует, что для вычисления универсальной весовой функции (3.5) достаточно вычислить проекцию

$$
P^{+}\left(\prod_{1 \leqslant \ell \leqslant n_{1}}^{\longrightarrow} f_{1}\left(t_{\ell}^{1}\right) \overline{\operatorname{Sym}}_{\bar{t}_{\bar{k}}^{2}}^{k_{2}^{1}}\left(P^{-}\left(\prod_{3 \leqslant i \leqslant N+1}^{\longrightarrow}\left(\prod_{k_{2}^{1}-k_{i-1}^{1}}^{\longrightarrow} f_{2 \leqslant j \leqslant k_{2}^{1}-k_{i}^{1}} f_{2, i}\left(t_{j}^{2}\right)\right)\right)\right)\right) .
$$

ПреДЛОЖЕНИЕ 3. Элемент, заданный проекцией (4.6), не равен нулю, только если

$$
n_{1}=k_{1}^{1} \geqslant k_{2}^{1}
$$

и может быть записан в виде

$$
\overline{\operatorname{Sym}}_{\bar{t}_{\bar{k}}^{1}, \bar{t}_{\bar{k}^{1}}}^{k_{1}^{1}, k_{2}^{1}}\left(\frac{1}{\left(k_{1}^{1}-k_{2}^{1}\right) !} Y\left(q^{-1} \tilde{t}_{\bar{k}^{1}}^{1} ; \bar{t}_{\bar{k}^{1}}^{2}\right) P^{+}\left(\prod_{2 \leqslant i \leqslant N+1}^{\longrightarrow}\left(\prod_{k_{1}^{1}-k_{i-1}^{1}} \prod_{1 \leqslant j \leqslant k_{1}^{1}-k_{i}^{1}} f_{1, i}\left(t_{j}^{1}\right)\right)\right)\right) .
$$

Здесь мы опять положили $k_{N+1}^{1}=0$. Комбинируя утверждения предложений 2 и 3 , заключаем, что результат коммутирования произведения токов $f_{1}\left(t_{1}^{1}\right) \ldots f_{1}\left(t_{n_{1}}^{1}\right)$ и элемента (4.3) может быть записан как $q$-симметризация по переменным $t_{1}^{1}, \ldots$ $\ldots, t_{n_{1}}^{1}, \ldots, t_{1}^{i}, \ldots, t_{k_{i}^{1}}^{i}, i=2, \ldots, N$, элемента $W^{1, N}\left(\bar{t}_{\bar{k}^{1}}\right)$.

Напомним, что разложение элемента (4.2) содержит $q$-симметризацию по переменным $t_{1}^{i}, \ldots, t_{n_{i}}^{i}, i=2, \ldots, N$. Это означает, что $q$-симметризация по переменным $t_{1}^{i}, \ldots, t_{k_{i}^{1}}^{i}, i=2, \ldots, N$, дает произведение факториалов $\prod_{i=2}^{N} k_{i}^{1}$ ! благодаря свойству $q$-симметричных функций (3.8). Это произведение сокращается с частью факториалов, которые возникают при разложении элемента (4.2) (см. предложение 1).

Суммируя сказанное, мы можем заключить, что универсальная весовая функция удовлетворяет следующему рекуррентному соотношению относительно ранга алгебры.

ПРЕДЛОЖЕНИЕ 4. Имеет место равенство

$$
\mathcal{W}^{1, N}\left(\bar{t}_{\bar{n}}\right)=\overline{\operatorname{Sym}}_{\bar{t}_{\bar{n}}} \sum_{\bar{k}^{1}}\left(\prod_{m=1}^{N} \frac{1}{\left(k_{m}^{1}-k_{m+1}^{1}\right) !} \prod_{m=2}^{N} \frac{1}{\left(n_{m}-k_{m}^{1}\right) !} \times\right.
$$




$$
\left.\times Z_{\bar{l}^{2}}^{2, N}\left(\bar{t}_{\bar{m}^{2}, \bar{n}^{2}}\right) W^{1, N}\left(\bar{t}_{\bar{k}^{1}}\right) \cdot \mathcal{W}^{2, N}\left(\bar{t}_{\bar{l}^{2}+\overline{1}, \bar{n}^{2}}\right)\right),
$$

где $k_{N+1}^{1}=0$.

Напомним, что в соответствии с нашими обозначениями $\bar{l}^{1}=\{0, \ldots, 0\}$ и $\hat{t}_{\bar{l}^{1}, \bar{k}^{1}} \equiv$ $\bar{t}_{\bar{k}^{1}}$. Другие наборы целых чисел, нумерующих коллекции переменных в формуле (4.9) и ассоциированные с подсистемой корней $\Pi_{2, N+1}$, суть

$$
\begin{aligned}
\bar{n}^{2} & =\left\{n_{2}, \ldots, n_{N}\right\}, & \bar{m}^{2} & =\{1, \ldots, 1\}, \\
\bar{l}^{2}+\overline{1} & =\left\{k_{2}^{1}+1, \ldots, k_{N}^{1}+1\right\}, & \bar{n}^{2} & =\left\{n_{2}, \ldots, n_{N}\right\} .
\end{aligned}
$$

Для завершения доказательства теоремы 1 необходимо воспользоваться предложением 4 несколько раз, представляя каждый раз универсальную весовую функцию для меньшей алгебры как $q$-симметризацию по соответствующему набору переменных и используя свойство (3.8) q-симметричных функций для сокращения лишнего произведения факториалов.

\section{ПРИЛОЖЕНИЕ}

Для доказательства предложения 2 достаточно доказать предложение 3 в несколько более общем случае. Пусть $\bar{k}_{i, j}=\left\{k_{i} \geqslant k_{i+1} \geqslant \cdots \geqslant k_{j} \geqslant 0\right\}$ - набор положительных невозрастающих целых чисел и $\bar{k}_{i+1, j}=\left\{k_{i+1} \geqslant k_{i+2} \geqslant \cdots \geqslant k_{j} \geqslant 0\right\}$ - поднабор этого набора. Пусть $\bar{t}^{i}=\left\{t_{1}^{i}, \ldots, t_{k_{i}}^{i}\right\}$ и $\bar{t}^{i+1}=\left\{t_{1}^{i+1}, \ldots, t_{k_{i+1}}^{i+1}\right\}$ - два набора переменных. Обозначим подалгебру в $U_{f}$, отвечающую набору корней $\Pi_{i, j+1}=\left\{\alpha_{i}, \ldots, \alpha_{j}\right\}$, как $U_{i, j}$.

ЛЕмма. Определенные в (2.19) экранирующие операторы удовлетворяют следующим свойствам:

$$
\begin{gathered}
f_{i, j}(t)-S_{f_{j-1, j}[0]}\left(f_{i, j-1}(t)\right)=0 \quad\left(\bmod P^{-}\left(U_{j-1, j-1}\right) \cdot U_{i, j-i}\right) ; \\
S_{f_{j-1, j}[0]}^{2}\left(f_{i, j-1}(t)\right)=0 ;
\end{gathered}
$$

экранирующие операторь коммутируют с проекциями $P^{+} u P^{-}$.

Доказательство этой леммы приведено в работах [5], [9]. Отметим, что (П.1) эквивалентно приведенному ниже соотношению (П.8) и определению сложных токов как сдвинутых произведений (2.16). Свойство (П.2) является следствием соотношений Ceppa (2.7).

ПреДЛОЖЕНИЕ 5. Произведение струны $F_{\bar{k}_{i, i+1}}\left(\bar{t}^{i}\right)$ и q-симметризации отрицательной проекции струны $P^{-}\left(F_{\bar{k}_{i+1, j}}\left(\bar{t}^{i+1}\right)\right)$ может быть записано в виде

$$
\begin{aligned}
& F_{\bar{k}_{i, i+1}}\left(\bar{t}^{i}\right) \cdot \overline{\operatorname{Sym}}_{\bar{t}^{i+1}}^{k_{i+1}}\left(P^{-}\left(F_{\bar{k}_{i+1, j}}\left(\bar{t}^{i+1}\right)\right)\right)= \\
& \quad=\frac{1}{\left(k_{i}-k_{i+1}\right) !} \overline{\operatorname{Sym}}_{\bar{t}^{i}, \bar{t}^{i+1}}^{k_{i+1}}\left(Y\left(q^{-1} \tilde{t}^{i}, \bar{t}^{i+1}\right) F_{\bar{k}_{i, j}}\left(\bar{t}^{i}\right)\right) \quad\left(\bmod P^{-}\left(U_{i+1, j}\right) \cdot U_{i, i}\right),
\end{aligned}
$$


где $\tilde{t}^{i}=\left\{t_{k_{i}-k_{i+1}+1}^{i}, \ldots, t_{k_{i}}^{i}\right\}$ есть поднабор набора $\bar{t}^{i}$.

ДокАЗАТЕЛЬСТво основано на соотношении, рассмотренном в подробностях в работе [9]:

$$
\begin{aligned}
& F_{\bar{k}_{i, i+1}}\left(\bar{t}^{i}\right) \cdot \overline{\operatorname{Sym}}_{\bar{t}^{i+1}}^{k_{i+1}}\left(P^{-}\left(F_{\bar{k}_{i+1, i+2}}\left(\bar{t}^{i+1}\right)\right)\right)= \\
& \quad=\frac{1}{\left(k_{i}-k_{i+1}\right) !} \overline{\operatorname{Sym}}_{\bar{t}^{i}, \bar{t}^{i+1}}^{k_{i+1}, k_{i+1}}\left(Y\left(q^{-1} \tilde{t}^{i}, \bar{t}^{i+1}\right) F_{\bar{k}_{i, i+2}}\left(\bar{t}^{i}\right)\right) \quad\left(\bmod P^{-}\left(U_{i+1, i+1}\right) \cdot U_{i, i}\right) .
\end{aligned}
$$

Мы не будем вдаваться в детали доказательства формулы (П.4), заметим лишь, что оно существенным образом опирается на правило коммутирования полного тока и отрицательной проекции тока, отвечающей простым корням,

$$
\begin{aligned}
f_{i, i+1}(t) P^{-} & \left(f_{i+1, i+2}(s)\right)=\frac{1}{1-q s / t} f_{i, i+2}(t)+ \\
& +\left(\frac{q-s / t}{1-q s / t} P^{-}\left(f_{i+1, i+2}(s)\right)+\frac{q^{-1}-q}{1-q s / t} P^{-}\left(f_{i+1, i+2}\left(q^{-1} t\right)\right)\right) f_{i, i+1}(t),
\end{aligned}
$$

которое является частным случаем (П.4) для $k_{i}=k_{i+1}=1$ при отождествлении $t_{1}^{i}=t, t_{1}^{i+1}=s$. Заметим, что соотношение (П.5) является прямым следствием формулы (2.14) для $s=i, j=i+2$.

Утверждение предложения 5 может быть получено из формулы (П.4) при применении последовательности экранирующих операторов (2.19)

$$
S_{\bar{k}_{i+2, j}} \stackrel{\text { def }}{=} S_{f_{j-1, j}[0]}^{k_{j}} \cdot S_{f_{j-2, j-1}[0]}^{k_{j-1}} \ldots S_{f_{i+3, i+4}[0]}^{k_{i+3}} \cdot S_{f_{i+2, i+3}[0]}^{k_{i+2}} \cdot
$$

При этом следует воспользоваться свойствами экранирующих операторов, сформулированными в лемме. Завершая доказательство, применим соотношение

$$
\begin{aligned}
S_{f_{l+1, l+2}[0]}^{k_{l+1}} & \left(\overline{\operatorname{Sym}}_{\bar{t}^{i}}^{k_{i}}\left(F_{\bar{k}_{i, l}}\left(\bar{t}^{i}\right)\right)\right)= \\
= & \frac{k_{l} !}{\left(k_{l}-k_{l+1}\right) ! k_{l+1} !} \overline{\operatorname{Sym}}_{\bar{t}^{i}}^{k_{i}}\left(F_{\bar{k}_{i, l+1}}\left(\bar{t}^{i}\right)\right) \quad\left(\bmod P^{-}\left(U_{l+1, l+1}\right) \cdot U_{i, l}\right),
\end{aligned}
$$

которое является следствием равенства (2.17) для $s=j-2$

$$
f_{i, j}(t)=S_{f_{j-1, j}[0]}\left(f_{i, j-1}(t)\right)+\left(q^{-1}-q\right) P^{-}\left(f_{j-1, j}\left(q^{i-j+1} t\right)\right) f_{i, j-1}(t)
$$

и коммутационных соотношений между сложными токами.

Утверждение предложения 3 следует непосредственно из применения проекции $P^{+}$к соотношению (П.3), взятому при $i=1$ и $j=N$. Для доказательства предложения 2 мы должны, используя (П.3), сгенерировать струну $F_{\bar{k}_{2, N}}\left(\bar{t}_{\bar{k}^{1}}^{2}\right)$ из произведения токов, отвечающих максимальному положительному корню $\alpha_{N}$ в соответствии с порядком (2.13).

Благодарности. Работа была поддержана INTAS (грант INTAS-OPEN-03-513350), программой Гейзенберг-Ландау, РФФИ (грант № 04-01-00642) и Программой поддержки ведущих научных школ (грант № НШ-8065.2006.2). 


\section{Список литературы}

[1] П. П. Кулиш, Н. Ю. Решетихин, ЖЭТФ, 80 (1981), 214.

[2] P. P. Kulish, N. Yu. Reshetikhin, J. Phys. A, 16 (1983), L591.

[3] А. Варченко, В. Тарасов, Алгебра и анализ, 6:2 (1994), 90.

[4] V. Tarasov, A. Varchenko, Astérisque, 246 (1997), 1.

[5] B. Enriquez, S. Khoroshkin, S. Pakuliak, Weight functions and Drinfeld currents, math.QA/0610398.

[6] В. Г. Дринфельд, Докл. АН СССР, 269 (1987), 13.

[7] B. Enriquez, V. Rubtsov, Israel J. Math., 112 (1999), 61.

[8] Д. Динг, С. З. Пакуляк, С. М. Хорошкин, ТМФ, 124:2 (2000), 179.

[9] С. З. Пакуляк, С. М. Хорошкин, ТМФ, 145:1 (2005), 3.

[10] S. Khoroshkin, S. Pakuliak, V. Tarasov, Off-shell Bethe vectors and Drinfeld currents, math.QA/0610517.

[11] V. Drinfeld, "Quantum groups", Proc. Int. Congr. Mathemacians (Berkeley, CA, 1986), ed. A. M. Gleason, Amer. Math. Soc., Providence, 1987, 798; M. Jimbo, Lett. Math. Phys., 10:1 (1985), 63.

[12] S. Khoroshkin, V. Tolstoy, Twisting of quantum (super)algebras. Connection of Drinfeld's and Cartan-Weyl realizations for quantum affine algebras, MPI Preprint MPI/94-23; hep-th/9404036.

[13] J. Ding, S. Khoroshkin, S. Pakuliak, Lett. Math. Phys., 53:2 (2000), 121. 